\title{
Fault Detection and Isolation for a Supermarket Refrigeration System - Part One: Kalman-Filter-Based Methods
}

\author{
Zhenyu Yang* Karsten B. Rasmussen ** Anh T. Kieu** \\ Roozbeh Izadi-Zamanabadi ${ }^{* * *}$ \\ * Department of Energy Technology, Aalborg University, Esbjerg \\ Campus, Niels Bohrs Vej 8, 6700 Esbjerg, Denmark (e-mail: \\ yang@et.aau.dk) \\ ** Former students of Intelligent Reliable Systems (IRS) Master \\ Program at Aalborg University, Denmark \\ *** Department RA-DT, Danfoss A/S, Nordborgvej 81, 6430 Nordborg, \\ Denmark (e-mail: Roozbeh@danfoss.com)
}

\begin{abstract}
:
Fault Detection and Isolation (FDI) using the Kalman Filter (KF) technique for a supermarket refrigeration system is explored. Four types of sensor fault scenarios, namely drift, offset, freeze and hard-over, are considered for two temperature sensors, and one type of parametric fault scenario, namely freeze-over/dirty built-up, is considered for one heat transfer coefficient between the inside air and the evaporator surface. For fault detection purpose, the fault residual is generated through a KF and then evaluated through CUSUM method. For fault isolation purpose, a bank of KFs arranged by splitting measurements is constructed for sensor fault isolation, while the Multi-Model Adaptive Estimation (MMAE) method is employed to handle parametric fault isolation. All these approaches are extended and checked by using Extended KF technique afterwards. The test results show that the EKF-based FDI method generally performances better and faster than the KF-based method does. However, both methods can not handle the isolation between sensor faults and parametric fault.
\end{abstract}

\section{INTRODUCTION}

There is no doubt that even a small improvement of efficiency and reliability of supermarket refrigeration systems can bring significant economic benefits in terms of saving foods values, reducing power consumption, and protecting the environment as well. An automatic monitoring system is often required to achieve this objective. The manual check of each display case in a big supermarket can be very time consuming and unreliable. Furthermore, there is the risk of a long delay time for finding a fault/failure.

A detailed supermarket refrigeration system model is developed in (Larsen et al. [2007] for control and monitoring study purpose. This model is a kind of prototype and it can be adapted to most current store-based refrigeration systems by adjusting some system parameters. The objective of this work is to investigate a Fault Detection and Isolation (FDI) solution for this considered system. There are four types of sensor fault scenarios, namely drift, offset, freeze and hard-over, need to be considered for two temperature sensors, and one type of parametric fault, namely freeze-over/dirty built-up, needs to be considered for a heat transfer coefficient between the inside air and evaporator surface.

The FDI technique has been extensively studied in recent decades, such as Blanke et al. [2003], Chen and Patton [1999], Frank and Ding [1997], Isermann [2006], Simani et al. [2003] and references therein. By reviewing most of available FDI methods, we decided to focus on stateestimation based FDI methods at our starting stage. The state-estimation can be observer-based methods (Frank and Ding [1997]), or Kalman Filter (KF)/Extended Kalman Filter (EKF) based methods (Chen and Patton [1999], Isermann and Balle [1997], Zhang and Li [1998]). A single observer/filter or a bank of observers/filters can be arranged for different FDI purpose (Isermann [2006]). In the following we will focus on the KF and EKF-based FDI methods for the considered refrigeration system. In our sister work (Yang et al. [2011]), an Unknown-Inpt-Observer (UIO) based method is studied and then compared with the KF and EKF methods proposed here. Finally, an EKF and UIO combined method is proposed as a complete FDI solution for the considered system in (Yang et al. [2011]).

The rest of the paper is organized in the following: Section 2 gives a brief overview of the considered system and potential fault scenarios; Section 3 explores the KF-based FDI and illustrate some results; Section 4 extends the same approach to EKF-based FDI method; and finally, we discuss and conclude the paper in Section 5.

\section{CONSIDERED SYSTEM MODEL AND FAULT SCENARIOS}

The considered refrigeration system, proposed in (Larsen et al. [2007]), consists of a number of sub-models, namely compressor, condenser, evaporator and expansion valve, 


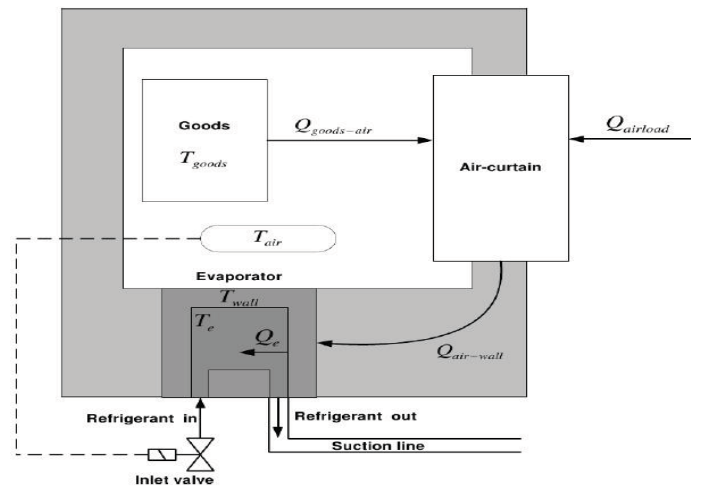

Fig. 1. Sketched Diagram of a Display Case (Larsen et al. [2007])

and a number of display cases. Some system variables/parameters which will be used in the following are listed in Table 1.

\begin{tabular}{|c|c|l|}
\hline Variable & Unit & Description \\
\hline$T_{\text {sub }}$ & $C$ & Temp. of the subscript $s u b$ \\
\hline$M_{\text {refrig }}$ & $k g$ & Mass of refrigerant in evaporator \\
\hline$P_{\text {suc }}$ & $b a r$ & Pressure in suction manifold \\
\hline$V_{p}$ & $N / A$ & Pos. of exp. valve, $0=$ closed, $1=$ open \\
\hline$Q_{a i r l o a d}$ & $J$ & Heat Flow from ambient air \\
\hline$M_{\text {sub }}$ & $k g$ & Mass of the subscript $s u b$ \\
\hline$C_{P_{s u b}}$ & $\frac{J}{k g \cdot K}$ & Specific heat capacity of $s u b$ \\
\hline$U A_{s u b_{a} \rightarrow s u b_{b}}$ & $\frac{J}{s \cdot K}$ & Heat transfer coeff. from $s u b_{a}$ to $s u b_{b}$ \\
\hline$\dot{Q}_{s u b_{a} \rightarrow s u b_{b}}$ & $\frac{J}{s}$ & Energy flow rate from $s u b_{a}$ to $s u b_{b}$ \\
\hline
\end{tabular}

Table 1. System Parameters and Variables

\subsection{Considered System Model}

The current focus of the considered system is on the dynamics relevant to display cases. As shown in Figure 1 , a general display case model consists of the evaporator and expansion valve parts. Moreover, their dynamics are also affected by both the air temperature and goods temperature inside the cases, the walls between the evaporator and display-area. A four-state nonlinear model of these concerned parts is summarized as (Larsen et al. [2007]):

$$
\begin{aligned}
& \dot{T}_{\text {goods }}=-\frac{U A_{\text {ga }}}{M_{\text {goods }} C_{P_{\text {goods }}}} T_{\text {goods }}+\frac{U A_{\text {ga }}}{M_{\text {goods }} C_{P_{\text {goods }}}} T_{\text {air }} \\
& \dot{T}_{\text {air }}=\frac{U A_{g a}}{M_{a i r} C_{P_{a i r}}} T_{\text {goods }}-\frac{U A_{g a}+U A_{a w}}{M_{a i r} C_{P_{a i r}}} T_{a i r} \\
& +\frac{U A_{\text {aw }}}{M_{\text {air }} C_{P_{\text {air }}}} T_{\text {wall }}+\frac{\dot{Q}_{\text {airload }}}{M_{\text {air }} C_{P_{\text {air }}}} \\
& \dot{T}_{\text {wall }}=\frac{U A_{\text {aw }}}{M_{\text {wall }} C_{P_{\text {wall }}}} T_{\text {air }}-\frac{U A_{\text {aw }}+U A_{\text {wr }} \frac{M_{\text {refrig }}}{M_{\text {refrigmax }}}}{M_{\text {wall }} C_{P_{\text {wall }}}} T_{\text {wall }}
\end{aligned}
$$

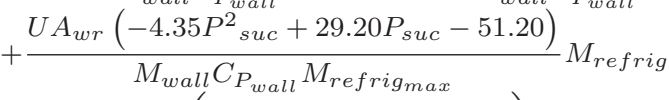

$$
\begin{aligned}
& \dot{M}_{\text {refrig }}=V_{p}\left(\frac{M_{\text {refrigmax }}-M_{\text {refrig }}}{\tau_{\text {fill }}}\right)+\left(V_{p}-1\right) \\
& \left(\frac{U A_{w r} \frac{M_{\text {refrig }}}{M_{\text {refrigmax }}}\left(T_{\text {wall }}+4.35 P_{\text {suc }}^{2}-29.22 P_{\text {suc }}+51.20\right)}{\left(0.022 P_{\text {suc }}^{2}-0.17 P_{\text {suc }}+2.30\right) \cdot 10^{5}}\right)
\end{aligned}
$$

where $U A_{w r} \hat{=} U A_{\text {wall } \rightarrow \text { refrigmax }}, U A_{\text {ga }} \hat{=} U A_{\text {goods } \rightarrow \text { air }}$ and $U A_{a w} \hat{=} U A_{a i r \rightarrow \text { wall }}$.

\subsection{Fault Scenarios}

Four type of fault scenarios are considered for two temperature sensors, i.e., $T_{\text {air }}$ and $T_{\text {wall }}$. The sensor fault scenarios are Drift, offset, freeze and hard-over. One type of parametric fault is considered regarding the heat transfer coefficient $U A_{\text {air } \rightarrow \text { wall, namely ice/dirt buildup on the }}$ evaporator.

Sensor Drift A drifting sensor will often over time converge to a specific value (i.e. zero or a sensor minimum or maximum). The drift fault can be hard to detect as the dynamics only change marginally. The slope of the drift determines how hard it is to be detected and isolated. In this study, a ramp with a slope of $+0.001{ }^{\circ} \mathrm{C}$ is added to the temperature measurement per sample.

Sensor Offset The offset error adds or subtracts a fixed value or percentage of the real value to all measurements. Here it is simulated as a constant which is added to or subtracted from the measurement. For instance, a fixed value of $2.5^{\circ} \mathrm{C}$ is added to each measurement.

Sensor Freeze When a sensor freezes, it stops making new measurements at any given point, often because of mechanical restriction. An example could be a sensor which is mechanically hindered from moving, and therefore gives the same measurement at all samples. In this study, the sensor freeze is simulated by repeating the last given signal.

Sensor Hard-Over The hard-over fault has some resemblance to the freeze fault, but instead of stopping within the working range, it jumps to an extreme value. This will usually be the lower or upper boundary of the measurable range of the sensor or zero. The fault could come from a sensor dropping out, a short circuit, sensor power-loss and many similar problems. The consequent abrupt and large change in dynamics could make this type of fault easy to be detected. Here this type of fault is simulated by substituting the measured signals by a given constant, e.g., $25^{\circ} \mathrm{C}$.

Parametric Fault The parameter $U A_{\text {air } \rightarrow \text { wall governs }}$ the heat transfer between the air inside the display case and the evaporator wall. Two regularly occurring faults that will change the $U A_{\text {air } \rightarrow \text { wall }}$ are

- Freeze-over. This happens when the refrigeration system has been running for some time. The surface of the evaporator is prone to condensation, which combined with the low temperatures, will build up ice on the surface of the evaporator. When the surface of the evaporator is full of ice, the surface area gets smaller and thereby less efficient. Furthermore, there will be a delay in transferring the heat from the refrigerant to the air, as the heat transfer now needs to go through another medium.

- Dirt build-up. As a lot of air from the shop passes through the evaporator, given enough time, it will be covered in dust and dirt. As the surface is covered more and more, the airflow through the evaporator will be increasingly limited. The combination of less air flowing through the condenser and the extra 

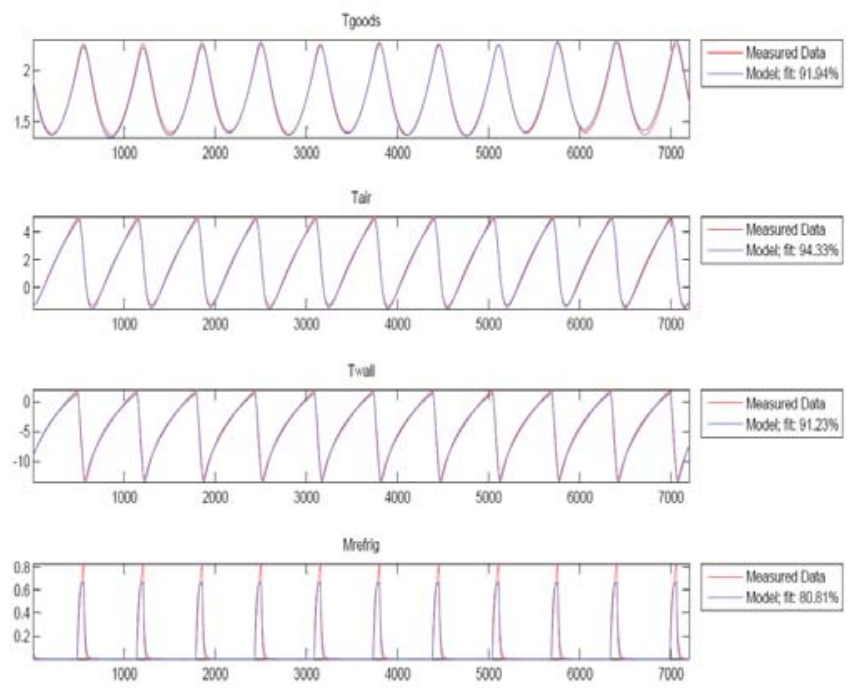

Fig. 2. Comparison of Linear Model and Measurements

medium (the dirt) which needs to be overcome, results in a reduced heat transfer coefficient.

In this study, the parametric fault is simulated as a steady drop in $U A_{\text {air } \rightarrow \text { wall }}$ from 500 at 6000 sec to 250 at time $16000 \mathrm{sec}$

\section{KALMAN-FILTER-BASED FDI}

A single KF based on a linearized nominal system is employed for fault detection purpose. While a bank of KFs arranged according to the splitting measurements method (Isermann [2006]) is constructed for the fault isolation purpose.

\subsection{Linearized Model}

A linear state space representation is derived by linearizing the nonlinear system (1), i.e.,

$$
\dot{x}(t)=A x(t)+B u(t)+E d(t), y(t)=C x+w(t),
$$

where the state $x$, control input $u$, disturbance $d$ are defined as

$$
x \hat{=}\left[\begin{array}{c}
T_{\text {goods }} \\
T_{\text {air }} \\
T_{\text {wall }} \\
M_{\text {refrig }}
\end{array}\right], u \hat{=}\left[\begin{array}{c}
V_{p} \\
P_{\text {suc }}
\end{array}\right], d \hat{=} Q_{\text {airload }}, y \hat{=}\left[\begin{array}{c}
T_{\text {air }} \\
T_{\text {wall }}
\end{array}\right] .
$$

$w(t)$ is the measurement noise. The equilibrium point is obtained by experimentally running the complete model (built up by Dymola software in Danfoss A/S). The comparison of the linearized system with original system is shown in Figure 2, it can be observed that there are over $90 \%$ fitness for all temperatures, and about $80 \%$ fitness of the mass flow. This linear model is further discretized before it is employed for a KF design.

\subsection{Detection Residual Generation}

We refer to (Shanmugan and Breipohl [1988]) for general Kalman filter theory. In order to keep the continuity of

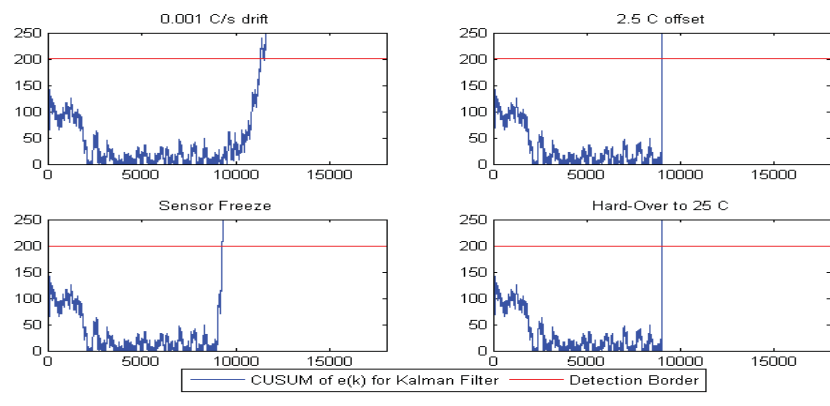

Fig. 3. KF-CUSUM for $T_{\text {air }}$ sensor faults
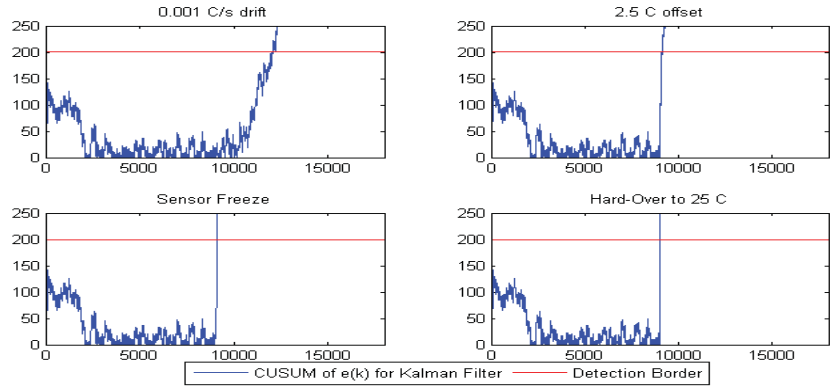

Fig. 4. KF-CUSUM for $T_{\text {wall }}$ sensor faults

this paper, the KF formulas (discrete-time) used here are summarized as:

$$
\left\{\begin{array}{l}
\hat{x}^{-}(k)=A_{k} \hat{x}(k-1)+B_{k} u(k-1) \\
P^{-}(k)=A_{k} P(k-1) A_{k}^{T}+Q_{k} \\
K(k)=P^{-}(k) C_{k}^{T} S^{-1}(k) \\
\hat{x}(k)=\hat{x}^{-}(k)+K(k)\left(y(k)-C_{k} \hat{x}^{-}(k)\right) \\
P(k)=\left(I-K(k) C_{k}\right) P^{-}(k)
\end{array}\right.
$$

where the innovation covariance $S(k)$ is

$$
S(k)=C_{k} P^{-}(k) C_{k}^{T}+R_{k} .
$$

The KF's innovation vector, which is defined as

$$
\operatorname{Res}(k)=y(k)-C_{k} \hat{x}^{-}(k),
$$

is used to construct a normalized residual based on (5) as:

$$
e_{k}=\operatorname{Res}^{T}(k) S^{-1}(k) \operatorname{Res}(k) .
$$

\subsection{Detection of System Fault}

In practice, detection is done by comparing the residual to a pre-determined threshold. In order to make the residual deviation more clear, a CUSUM (Cumulative Sum) method is used for residual evaluation (Basseville and Nikiforov [1993]), i.e.,

$$
Z(k)=\max \left(0, Z(k-1)+\left(e(k)-\omega_{n}\right)\right)
$$

where $\omega_{n}$ is the mean value of the residual under nominal operation, and $Z(0)=0$. The threshold is selected based on experiments. The results are illustrated in Figure 3 and 4 for sensor faults, respectively. While the results regarding to the parametric fault is shown in Figure 5. It can be observed that all concerned faults can be easily detected. 


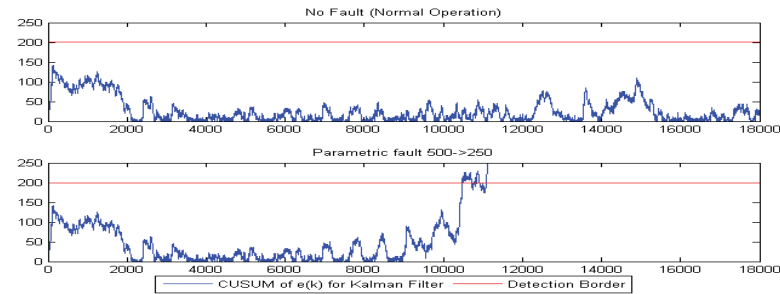

Fig. 5. KF-CUSUM for nominal and $U A_{\text {air } \rightarrow \text { wall }}$ drop (Dirt/Ice)

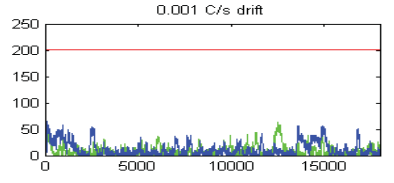

Sensor Freeze

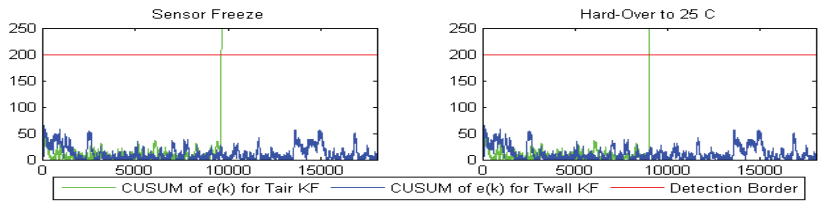

Fig. 6. KF-CUSUM Isolation for $T_{a i r}$ Sensor Faults

\subsection{Isolation of System Fault}

There are a number of methods can be used to localize where the detected fault has occurred. One popular approach is to develop a bank of observers/KFs, and each observer/filter uses only some specific measurement (not all of them). This approach is quite useful to handle potential sensor faults (Isermann [2006]). Another general approach is to employ the multiple-model techniques, and each model corresponds to one specific faulty/nominal scenario. This approach can handle more general situations, as long as all potential fault scenarios can be known beforehand (Isermann [2006],Zhang and Li [1998]). In the following, the first approach is investigated for isolating sensor faults, while the second approach is used for isolating the parametric fault and different operating conditions.

Isolation of Sensor Fault There are two general approaches to split measured signals for a bank of KFs:

- Using only one sensor measurement per filter, or

- Using all except one sensor measurement per filter.

As explained in Isermann [2006], the first approach can potentially isolate multiple fault scenarios, while the second method is good in its robustness to plant deviations. Since there are only two sensor measurements, both methods will lead to identical analysis here. Thereby, the KF bank developed here consists of only two KFs for $T_{a i r}$ and $T_{\text {wall }}$. Some test results are illustrated in Figure 6, 7 and 8, respectively, where the green line is the residual from the first $\mathrm{KF}$ using $T_{\text {air }}$ as input, and the blue line is from the second KF using $T_{\text {wall }}$ as input.

As can be seen from Figure 6 and 7, sensor freeze and hard-over can be quite easily detected. Unfortunately, the residual of sensor offset case only passed the threshold for a short while for $T_{\text {air }}$ scenario, but not at all for the $T_{\text {wall }}$ case. The isolation of sensor drift is impossible, at least for small changes in temperature. Nevertheless, the drop in
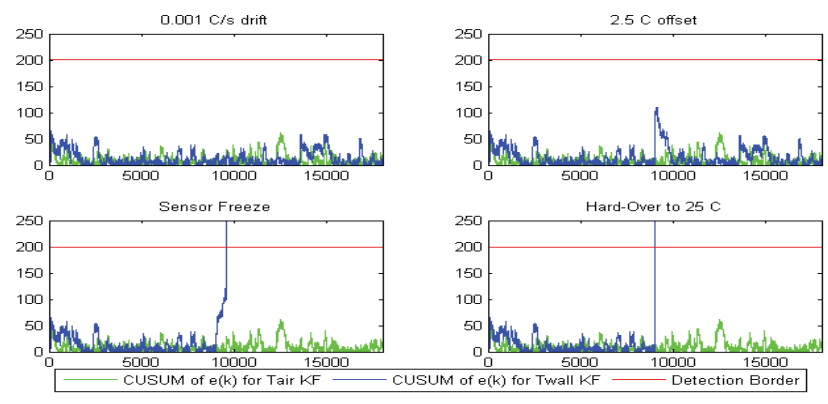

Fig. 7. KF-CUSUM Isolation for $T_{\text {wall }}$ Sensor Faults
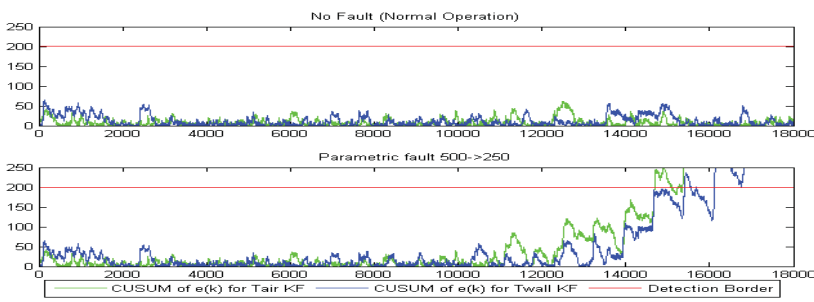

Fig. 8. CUSUM Isolation for nominal and $U A_{\text {air } \rightarrow \text { wall }}$ drop

$U A_{\text {air } \rightarrow \text { wall }}$ value (parametric fault) triggered a detection alarm with a claim that both sensors are faulty.

Isolation of Parametric Fault The multiple-model approach is investigated for parametric fault isolation. As the amount of goods inside the display case is allowed to change during the routine operation of the considered refrigeration system, a change in $M_{\text {goods }}$ should not be seen as a fault. Thereby, four different models are defined according to

(1) Full-load, no fault, i.e., $M_{\text {goods }}=200, U A_{\text {air } \rightarrow \text { wall }}=$ 500

(2) Non-full-load, no fault, $M_{\text {goods }}=25, U A_{\text {air } \rightarrow \text { wall }}=$ 500 ;

(3) Full-load, fault $\left(M_{\text {goods }}=200, U A_{\text {air } \rightarrow \text { wall }}=250\right.$; and

(4) Non-full-load, parametric fault, i.e., $M_{\text {goods }}=25$, $U A_{\text {air } \rightarrow \text { wall }}=250$.

Correspondingly, four KFs are constructed based on these model coefficients. The Multiple Model Adaptive Estimation (MMAE) (Zhang and Li [1998]) is employed here for selecting the most likely scenario in a online manner. The posterior probability of each model based on the latest residual information is calculated according to

$$
p(i \mid k)=\frac{l(i \mid k) p(i \mid k-1)}{\sum_{j=1}^{N} l(i \mid k) p(i \mid k-1)},
$$

where $N$ is the total amount of models, and $l(i \mid k)$ is the likelihood function of the $i$ 'th model at sample $k$ :

$$
l(i \mid k)=\frac{1}{\left|2 \pi S_{i}(k)\right|^{\frac{1}{2}}} \exp \left[-\frac{1}{2} \operatorname{Res}_{i}^{T}(k) S_{i}^{-1}(k) \operatorname{Res}_{i}(k)\right] .
$$

The parametric fault is introduced as a drift in $U A_{\text {air } \rightarrow \text { wall }}$ from 500 at $6000 \mathrm{sec}$ with a slope of $-250 / 10000$ down to 250 at $16000 \mathrm{sec}$, and this value is stable at 250 for the remaining time. As shown in Figure 9 and 10, the MMAE solution is not quite clean, but it still gave a clear and correct estimation of the corresponding model. However, 


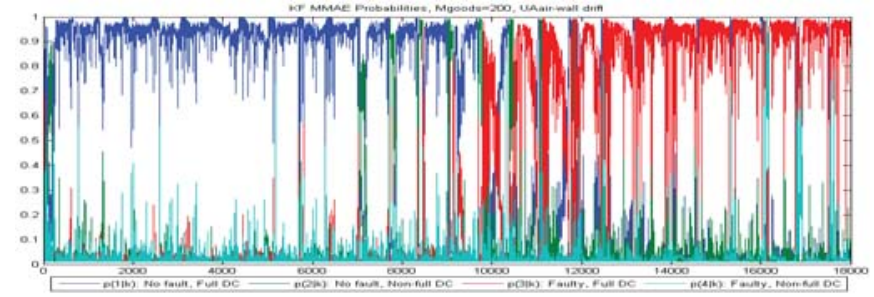

Fig. 9. KF-MMAE: $M_{\text {goods }}=200$, Drift in $U A_{\text {air } \rightarrow \text { wall }}$

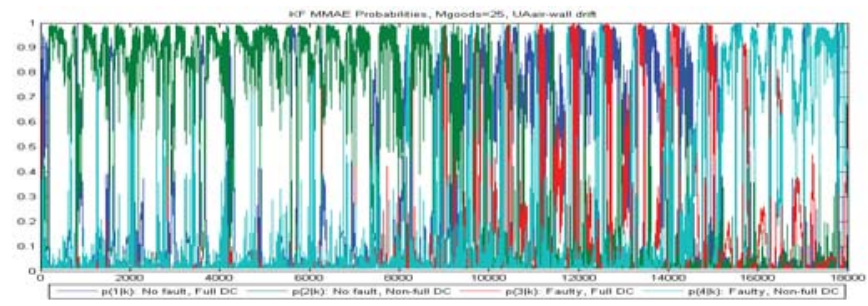

Fig. 10. KF-MMAE: $M_{\text {goods }}=25$, Drift in $U A_{\text {air } \rightarrow \text { wall }}$

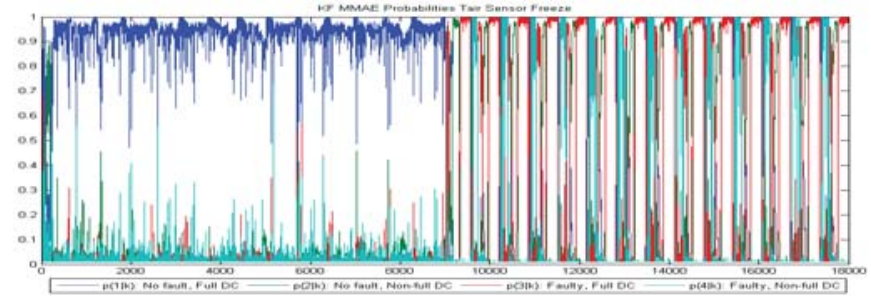

Fig. 11. KF-MMAE: $T_{\text {air }}$ Sensor Freeze
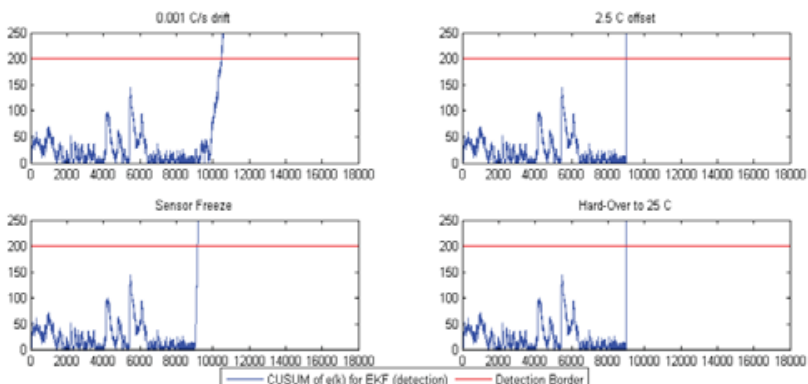

Fig. 12. EKF-CUSUM for $T_{a i r}$ Faults

if some sensor fault is introduced, the calculation (9) is no longer reliable. Figure 11 illustrates the situation where the $T_{\text {air }}$ sensor freezed at $9000 \mathrm{sec}$. This unexpected sensor fault made the MMAE claim a parametric fault, but not for sure about the load condition.

\section{EXTENDED-KF-BASED FDI}

In the following, all discussed FDI design and analysis are extended to use of EKF techniques. The motivation is quite clear: since the original system is nonlinear, how EKF-based method can improve the results derived from KF-based FDI method? The detection capability of a single EKF constructed based on (1) is illustrated in Figure 12, 13 and 14, respectively.

There is no doubt that the EKF can detect all concerned faults quickly. The study of fault isolation of sensor fault using a bank of EKFs is illustrated in Figure 15, 16 and 17 , respectively. It can be observed that, different

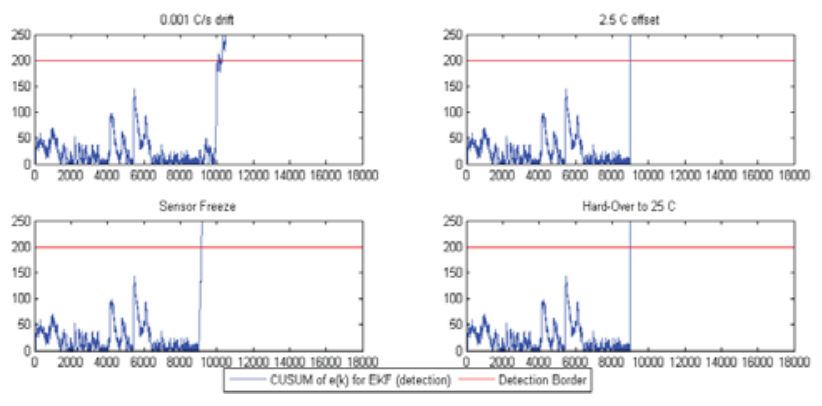

Fig. 13. EKF-CUSUM for $T_{\text {wall }}$ Faults

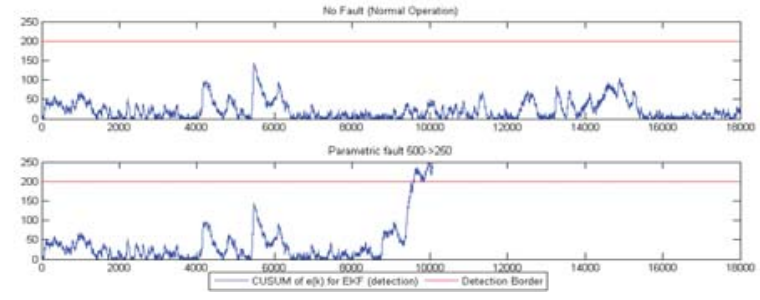

Fig. 14. EKF-CUSUM for Nominal and $U A_{\text {air } \rightarrow \text { wall }}$ Drop
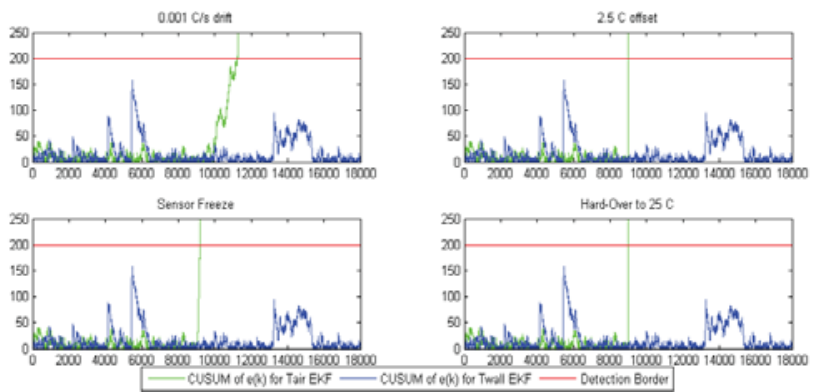

Fig. 15. EKF-CUSUM Isolation for $T_{\text {air }}$ Faults
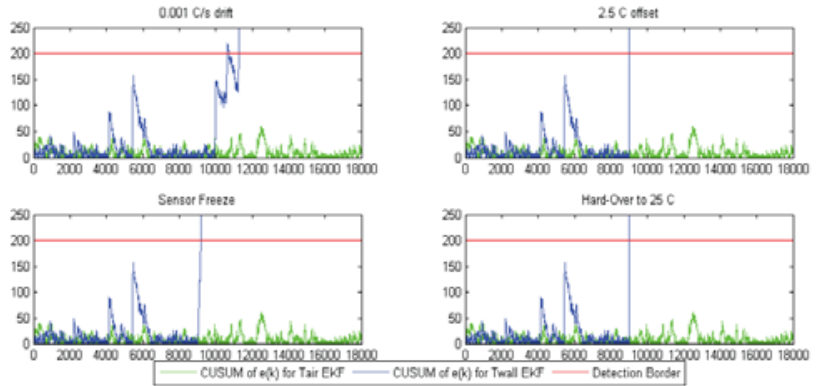

Fig. 16. EKF-CUSUM Isolation for $T_{\text {wall }}$ Faults

with the KF-based results, here all sensor fault scenarios can be distinguished clearly and quickly. Of course, the parametric fault still can cause a false claim $\left(T_{\text {air }}\right.$ fault) as shown in Figure 17.

The parametric fault isolation under different operating conditions, as assumed in previous section, are illustrated in Figure 19 and 18. It can be observed that the EKF-based methods give much more clear indications compared with KF-based methods.

\section{DISCUSSIONS AND CONCLUSION}

Both the KF and EKF-based methods can successfully detect all concerned faults correctly. If the parametric 


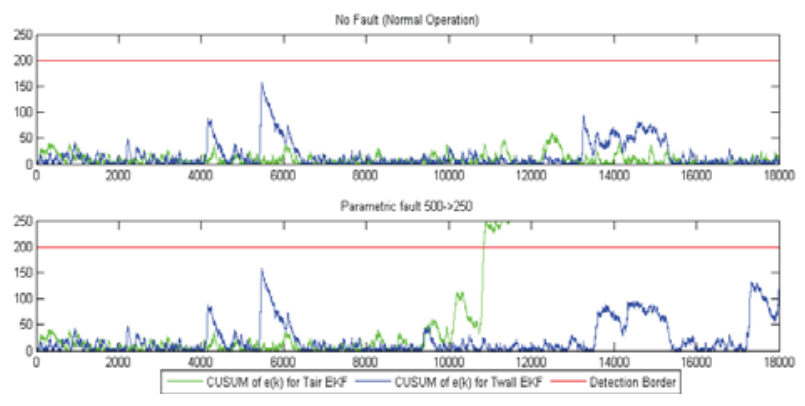

Fig. 17. EKF-CUSUM Isolation for $U A_{\text {air } \rightarrow \text { wall }}$ Drop

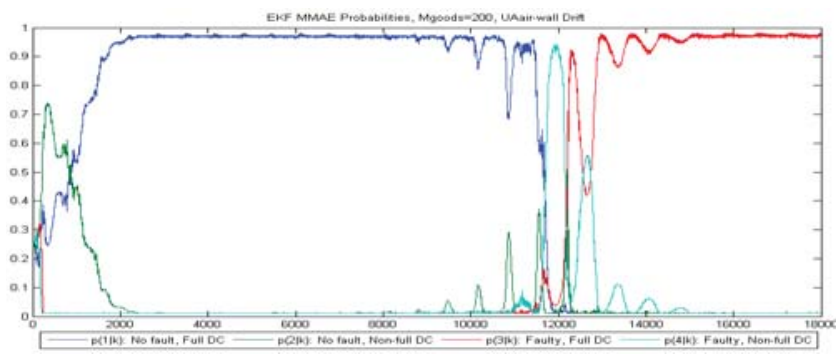

Fig. 18. EKF-MMAE: $M_{\text {goods }}=200$, Drift in $U A_{\text {air } \rightarrow \text { wall }}$

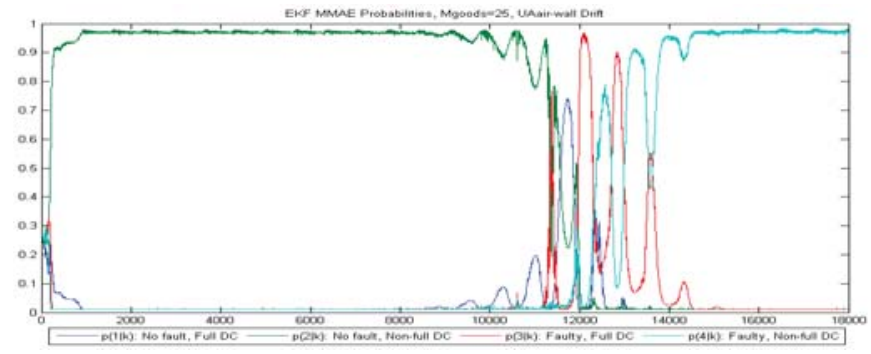

Fig. 19. EKF-MMAE: $M_{\text {goods }}=25$, Drift in $U A_{\text {air } \rightarrow \text { wall }}$

fault and sensor faults can be distinguished beforehand, a bank of EKFs can distinguish two different sensor faults, no matter what kind of fault scenarios. While KFbased method can only distinguish two sensor faults under freeze and hard-over scenarios. The parametric fault can be distinguished from the nominal operation under different operating conditions, no matter through KFbased MMAE method or EKF-based MMAE method. The EKF-based method gives faster and more clear indications than KF-based method does. In general, the EKFbased method provides better and faster results than KF method. However, the payoff for this benefit is that EKFbased method requires much more computation power (Yang et al. [2011]).

A critical limitation of the proposed methods is that both KF and EKF-based methods require the dynamic of state $T_{\text {goods }}$ and statistic feature of $Q_{\text {airload }}$ known. This assumption may be not true in reality. For instance, the amount and type of goods inside the display case can dynamically change in the store opening time, could due to the random customer's pickup or aperiodically supply delivery. The cover of the display case could be open or closed in a very random manner. However, if the goods condition inside the display case, the air temperature in the store and the display case cover status can be measured and informed the FDI system, some simple algorithm can

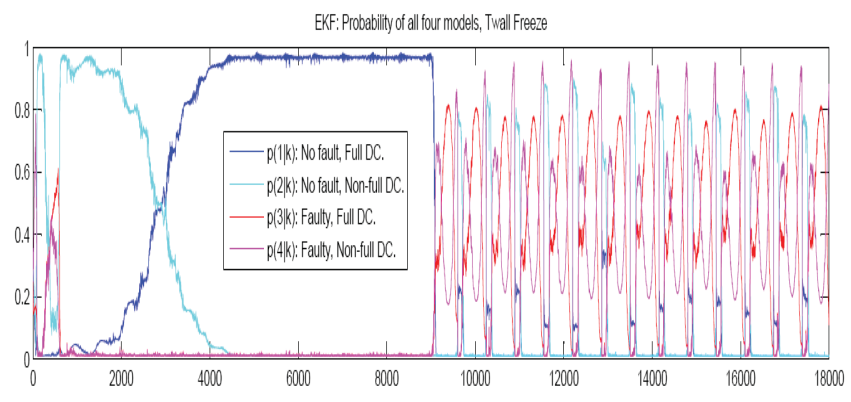

Fig. 20. EKF-MMAE Isolation, $T_{\text {wall }}$ Freeze Fault

be built up to estimate the $T_{\text {goods }}$ dynamic and statistic feature of $Q_{\text {airload }}$, In this way the EKF-based method can generally perform quite well and fast.

As we mentioned, whether we can have a successful FDI solution using the current KF- or EKF-based methods, depends on whether we can distinguish sensor faults from the parametric fault beforehand. As shown in Figure 20, a sensor fault can puzzle the EKF-based MMAE method, since this unexpected fault leads the operating system to be far away from any predefined model. It's clear that the current both methods can not handle this task. In our sister paper (Yang et al. [2011]), the Unknown-InputObserver (UIO)-based method is investigated for FDI design for the considered system. A bank of UIOs can be used to distinguish the parametric fault from sensor faults. After an extensive comparison of these KF-, EKFand UIO-based methods, a complete FDI solution by combining EKF-based MMAE and UIO-based methods is proposed in (Yang et al. [2011]). The complete FDI solution can detect and isolate all concerned faults in a reasonably fast, efficient and reliable manner.

\section{REFERENCES}

M. Basseville and I.V. Nikiforov. Detection of Abrupt Changes: Theory and Application. Prentice-Hall, Englewood Cliffs, 1993.

M. Blanke, M. Kinnaert, J. Lunze, and M. Staroswiecki. Diagnosis and fault-tolerant control. Springer, 2003.

J. Chen and R.J. Patton. Robust Model-Based Fault Diagnosis for Dynamic Systems. Kluwer Academic Publishers, 1999.

P.M. Frank and X. Ding. Survey of robust residual generation and evaluation methods in observer-based fault detection systems. $J$. Proc. Cont., 7(6):403-424, 1997.

R. Isermann. Fault-diagnosis systems - An introduction from fault detection to fault tolerance. Springer Verlag, Berlin, 2006.

R. Isermann and P. Balle. Trends in the application of modelbased fault detection and diagnosis of technical processes. Control Engineering Practice, 5:707-719, 1997.

L.F.S. Larsen, R. Izadi-Zamanabadi, and R. Wisniewski. Supermarket refrigeration system - benchmark for hybrid system control. In Proc. of ECC, Kos, Greece, Jul 2007.

K.S. Shanmugan and A.M. Breipohl. Random Signals: Detection, Estimation and Data Analysis. John Wiley \& Sons, 1988.

S. Simani, C. Fantuzzi, and R.I. Patton. Model-based fault diagnosis in dynamic systems using identification techniques. SpringerVerlag, 2003.

Z. Yang, K.B. Rasmussen, A.T. Kieu, and R. Izadi-Zamanabadi. Fault detection and isolation for a supermarket refrigeration system - part two: Unknown-input-observer method and its extension. Accepted by 18th IFAC World Congress, Milano, Italy, Aug.28-Sept.02 2011.

Y.M. Zhang and X.R. Li. Detection and diagnosis of sensor and actutor failures using imm estimator. IEEE Trans. Aerospace and Electronic Systems, 34(4):1293-1313, Oct. 1998. 